\title{
A lattice theory for low energy fermions at nonzero chemical potential
}

\author{
Jiunn-Wei Chen* \\ Department of Physics and NCTS at Taipei, National Taiwan University, Taipei 10617, Taiwan \\ and Department of Physics, CTP, Massachusetts Institute for Technology, Cambridge, MA 02139, USA \\ David B. Kaplan耳 \\ Institute for Nuclear Theory, University of Washington, Seattle, WA 98195-1550, USA
}

\begin{abstract}
We construct a lattice theory describing a system of interacting nonrelativistic spin $s=\frac{1}{2}$ fermions at nonzero chemical potential. The theory is applicable whenever the interparticle separation is large compared to the range of the two-body potential, and does not suffer from a sign problem. In particular, the theory could be useful in studying the thermodynamic limit of fermion systems for which the scattering length is much larger than the interparticle spacing, with applications to realistic atomic systems and dilute neutron gases.

PACS numbers: 71.10.Fd,74.20.Fg,03.75.Kk
\end{abstract}

\section{INTRODUCTION}

In this Letter we consider dilute fermion systems with attractive interactions, for which the effective range for two-body scattering is much less than the inter-particle spacing. Such systems are characterized by a dimensionless number $\eta=\left(a n^{1 / 3}\right)$, where $a$ is the two-body scattering length, and $n$ is the density. Such systems are well known to exhibit fascinating nonperturbative behavior. For $\eta$ small and negative (weak attraction) one finds the BCS solution with pairing and superconductivity. With $\eta$ small and positive, corresponding to strong attraction with a two-body bound state well below threshold, the bound pairs will Bose-Einstein condense. In each of these cases the behavior is nonperturbative, but since the effective interaction is weak, the system can be successfully described in a mean field approximation. On the other hand, dilute fermion systems for which the parameter $|\eta|$ is large are not amenable to analytical treatment. Physical realizations include both dilute neutron gases (the neutron-neutron scattering length is more than an order of magnitude greater than its effective range) as well as cold, dilute gases of fermionic atoms tuned to be near a Feshbach resonance 1, 2, 3]. Recently there has been intense interest in exploring such systems experimentally [4]. In the limit that $|\eta| \rightarrow \infty$ one expects to see universal behavior, so that the same dimensionless physical constants will apply equally to the atomic and nuclear systems.

Because of the prospects of exploring ultracold fermionic atoms at large $|\eta|$ experimentally, it is of great interest to understand such systems theoretically, which necessitates numerical studies. A recent numerical study of dilute fermions at large $|\eta|$ is found in Ref. [5]. The authors of that work used the fixed-node Diffusion Monte

\footnotetext{
*Electronic address: jwc@phys.ntu.edu.tw

${ }^{\dagger}$ Electronic address: dbkaplan@phys.washington.edu
}

Carlo method to extract the parameter $\xi$, defined as the energy per particle relative to the value for a noninteracting Fermi gas. Since this approach is variational, the result $\xi=0.44 \pm .01$ obtained is an upper bound on the true quantity.

The calculation [5] is performed at fixed particle number $N$, up to $N=38$. It is desirable to have a numerical approach which is not variational, and which can probe thermodynamic properties of dilute fermion gases, such as the critical temperature for the pairing transition. In this Letter we propose such a method, in which the fermions live on a spacetime lattice at nonzero chemical potential. We exploit the fact that all relevant scattering information at low density is contained in the scattering length, so that the complicated two-body interactions can all be replaced by an effective field theory with a single attractive four-fermion interaction. While lattice formulations of interacting fermions at finite density typically encounter a "sign problem", we show that is not the case here. There is another issue that requires attention, however, relating to possible instabilities introduced by a purely attractive interaction. We perform an analytical calculation that suggests such instabilities may be avoided.

\section{THE LATTICE FORMULATION OF THE EFFECTIVE THEORY}

To construct a lattice version of the continuum theory, we first analytically continue to Euclidean spacetime, represented by an $N_{\tau} \times N_{s}^{3}$ lattice, where $N_{\tau}$ and $N_{s}$ are the number of sites in the time and space directions respectively. We set $\hbar=1$ and measure all dimensionful quantities in units of the lattice spacing, which is taken to be the same in the space and time directions. We impose periodic boundary conditions in the space directions and anti-periodic boundary conditions in the time direction. This allows the Euclidean path integral to be interpreted as the finite temperature partition function, with inverse temperature $\beta=N_{\tau}$. The fermion fields at 
site $\mathbf{n}$ are denoted by $\psi_{\mathbf{n}}$ and $\bar{\psi}_{\mathbf{n}}$, and are independent, two-component Grassmann spinors.

The continuum Euclidean action for free fermions at nonzero chemical potential is $\int d^{4} x \bar{\psi}\left(\partial_{\tau}-\nabla^{2} / 2 M-\mu\right) \psi$. The corresponding lattice action is $S_{0}=\sum_{\mathbf{n}} \bar{\psi}_{\mathbf{n}}\left(K_{0} \psi\right)_{\mathbf{n}}$, where

$$
\left(K_{0} \psi\right)_{\mathbf{n}} \equiv\left(\psi_{\mathbf{n}}-e^{\mu} \psi_{\mathbf{n}-\hat{\mathbf{e}}_{\mathbf{0}}}\right)-\sum_{i=1}^{3} \frac{\left(\psi_{\mathbf{n}+\hat{\mathbf{e}}_{\mathbf{i}}}-2 \psi_{\mathbf{n}}+\psi_{\mathbf{n}-\hat{\mathbf{e}}_{\mathbf{i}}}\right)}{2 M}
$$

In these expressions $\hat{\mathbf{e}}_{\alpha}$ is a unit vector in the $\alpha$ direction ( $\alpha=0$ corresponding to Euclidean time $\tau$ ), $M$ is the fermion mass, and $\mu$ is the chemical potential which is treated like the time component of an imaginary gauge field. This follows the prescription in ref. [6] (except that we have the opposite sign for $\mu$ ) and ensures that the free energy does not have spurious cutoff dependence.

The free propagator computed from $S_{0}$ is

$$
G_{0}(\tau, \mathbf{p})=\frac{\xi_{\mathbf{p}}^{\tau}}{\left(1+\Delta_{\mathbf{p}} / M\right)\left(1+\xi_{\mathbf{p}}^{N_{\tau}}\right)} \times\left\{\begin{array}{ll}
1 & \text { if } \tau \geq 0 \\
-\xi_{\mathbf{p}}^{N_{\tau}} & \tau<0
\end{array},\right.
$$

where

$$
\xi_{\mathbf{p}} \equiv \frac{e^{\mu}}{1+\Delta_{\mathbf{p}} / M}, \quad \Delta_{\mathbf{p}}=2 \sum_{i=1}^{3} \sin ^{2} \frac{p_{i}}{2} .
$$

The time $\tau$ is an integer, corresponding to propagation by $\tau$ lattice spacings in the time direction, while the momentum components are given by $p_{i}=\hat{p}_{i} \frac{2 \pi}{N_{s}}$, with $\hat{p}_{i}$ being integers in the range $-N_{s} / 2<\hat{p}_{i} \leq N_{s} / 2$.

Note that for $\mu<0, \xi_{\mathbf{p}}<1$ and in the limit $N_{\tau} \rightarrow \infty$ (zero temperature) $\xi_{\mathbf{p}}^{N_{\tau}} \rightarrow 0$ and there is no propagation backward in time,

$$
G_{0}(\tau, \mathbf{p}) \underset{\mu<0, N_{\tau} \rightarrow \infty}{\longrightarrow} \frac{\xi_{\mathbf{p}}^{\tau} \theta(\tau)}{\left(1+\Delta_{\mathbf{p}} / M\right)},
$$

indicating the absence of antiparticles or holes in this nonrelativistic theory at zero density.

Fermion interactions can be represented by an effective field theory with a four-fermion interaction the strength of which is tuned to reproduce the physical two-body scattering length. On our lattice we generate the interaction by means of a non-propagating scalar auxiliary field $\varphi$ coupled to the fermions. By situating $\varphi$ along time-links of the lattice we can eliminate fermion loops lying on surfaces of constant Euclidean time, simplifying the analysis. Since one is interested in pairing correlations, it is convenient to also introduce a constant complex source $J$ for fermion pairs so that one can study the pairing transition at finite volume. These considerations lead us to the action

$$
\begin{aligned}
& S=\sum_{\mathbf{n}}\left[\bar{\psi}_{\mathbf{n}}(K \psi)_{\mathbf{n}}+\frac{1}{2} m^{2} \varphi_{\mathbf{n}}^{2}+\frac{1}{2}\left(J \psi_{\mathbf{n}} \sigma_{2} \psi_{\mathbf{n}}+\text { h.c. }\right)\right], \\
& (K \psi)_{\mathbf{n}} \equiv\left(K_{0} \psi\right)_{\mathbf{n}}-\varphi_{\mathbf{n}} e^{\mu} \psi_{\mathbf{n}-\hat{\mathbf{e}}_{0}} .
\end{aligned}
$$

An important property of this theory is that after integration over $\psi$ and $\bar{\psi}$, the remaining integral over $\varphi$ has positive semidefinite measure, so that Monte Carlo integration methods may be applied. This result is easy to see if the source $J$ is neglected, in which case integrating out the fermions yields a factor of $\operatorname{det} K . K$ is trivial in spin-space, and can be written as $K=\tilde{K} \otimes \mathbf{1}_{s}$ where $\mathbf{1}_{s}$ is the two dimensional identity matrix acting on spinor indices, and $\tilde{K}$ acts only in coordinate space. Thus $\operatorname{det} K=(\operatorname{det} \tilde{K})^{2}$ which is positive semidefinite, since $\tilde{K}$ is a real operator.

Including the constant source $J$, the fermionic action may be rewritten as $\frac{1}{2} \Psi^{T} \mathcal{A} \Psi$, where we have defined

$$
\Psi=\left(\begin{array}{c}
\psi \\
i \sigma_{2} \bar{\psi}^{T}
\end{array}\right), \quad \mathcal{A}=\left(\begin{array}{cc}
-i J & K^{\dagger} \\
K & -i J^{*}
\end{array}\right)\left(\begin{array}{cc}
i \sigma_{2} & 0 \\
0 & i \sigma_{2}
\end{array}\right) .
$$

Each block in this matrix is $2 N$ dimensional, where $N$ is the number of lattice sites; $K$ acts trivially on spinor indices, the Pauli matrix $\sigma_{2}$ acts trivially on coordinate indices, and $J$ is proportional to the identity matrix in both spaces. Note that $K^{T}=K^{\dagger}$ since $K$ is real. Integrating out the fermions yields a purely bosonic theory, where the path integral over the $\varphi$ field is weighted by $\exp \left(-\frac{m^{2}}{2} \sum_{\mathbf{n}} \varphi_{\mathbf{n}}^{2}\right)$ times the pfaffian of $\mathcal{A}$ :

$$
\operatorname{Pf}[\mathcal{A}]=\left|\begin{array}{cc}
-i J & \tilde{K}^{\dagger} \\
\tilde{K} & -i J^{*}
\end{array}\right|=\left.\sigma|| J\right|^{2}+\tilde{K}^{\dagger} \tilde{K} . \mid
$$

Each block in the above expression is $N$-dimensional. Thus $\operatorname{Pf}[\mathcal{A}]$ is real and positive semidefinite for all real values of $J, \mu$, and $\varphi_{\mathbf{n}}$, up to the irrelevant constant sign $\sigma=(-1)^{N}$, and there is no sign problem encountered with this lattice formulation. Similar analyses exist for two flavor QCD [7] and the NJL model [8].

We have introduced in Eq. (5) a new parameter $m^{2}$, which determines the strength of the two-body interaction. This parameter can be related to the two-body scattering length $a$ by summing the ladder diagrams with $\varphi$ exchange between the fermions at zero external momenta, zero temperature, infinite volume, and zero chemical potential, $\mu \rightarrow 0^{-}$; the result may be equated to $\frac{4 \pi a}{M}$ (see [9]). The result is

$$
\frac{m^{2}}{M}=-\frac{1}{4 \pi a}+L(M),
$$

where $L(M)$ is given by the integral

$$
\begin{aligned}
L(M) & =\int \frac{d^{3} \mathbf{p}}{(2 \pi)^{3}} \sum_{\tau=0}^{\infty}\left(G_{0}(\tau, \mathbf{p})\right)^{2} \\
& =\frac{1}{2} \int \frac{d^{3} \mathbf{p}}{(2 \pi)^{3}} \frac{1}{\Delta_{\mathbf{p}}+\Delta_{\mathbf{p}}^{2} / 2 M}
\end{aligned}
$$

where the integral is over the Brillouin zone, $\left|p_{i}\right| \leq \pi$.

Note that since $m^{2}$ must be positive, scattering lengths satisfying $\frac{1}{a}>4 \pi L(M)$ are inaccessible in our theory without introducing an imaginary coupling for $\varphi$ and sacrificing positivity of the measure. These values for the 
scattering length correspond to two-body bound states with $O(1 / M)$ binding energy in lattice units, which are not of physical interest in any case.

\section{PHASE STRUCTURE OF THE LATTICE THEORY}

An interacting continuum limit of our lattice theory requires that we be able to find a point in the phase diagram where both the interparticle spacing $n^{-1 / 3}$ and the scattering length $a$ diverge in lattice units. It does not require that the mass $M$ vanish, however. From Eq. (8) we see it is always possible to tune the parameter $m^{2}$ so that $a$ diverges. What is less obvious is that we can then choose $\mu$ so that the particle density $n$ becomes arbitrarily small. In general one would expect to find a line of first order phase transitions in the $\left(M, \frac{1}{a}\right)$ plane for sufficiently large values of $\frac{1}{a}$, corresponding to a strongly attractive four-fermion interaction. This follows from the well-known result in the continuum that a fermion system at fixed chemical potential with a purely attractive interaction has no ground state, being unstable against the formation of infinitely dense matter. On the lattice, where the maximum attainable density is limited, one would expect that at $\mu=0$ the ground state of the system would jump from $n=0$ to $n=O(1)$ in lattice units for a sufficiently strong attraction. The existence of a continuum limit then becomes whether for any mass $M$ the $n=0$ phase at $\mu=0$ persists when $1 / a \rightarrow 0$.

Therefore the first thing to compute in lattice theory is the relation between the chemical potential $\mu$ and the fermion density $n$. For $n$ we take

$$
\begin{aligned}
n & =\left\langle\bar{\psi}_{\mathbf{n}} e^{\mu} \psi_{\mathbf{n}-\hat{\mathbf{e}}_{\mathbf{0}}}\right\rangle=m^{2}\left\langle\varphi_{\mathbf{n}}\right\rangle \\
& =\frac{\int[d \varphi] \mathcal{P}(\varphi)\left(m^{2} \varphi_{\mathbf{n}}\right)}{\int[d \varphi] \mathcal{P}(\varphi)}
\end{aligned}
$$

where $\mathcal{P}(\varphi)=\left(e^{-\frac{m^{2}}{2} \sum_{\mathbf{n}} \varphi_{\mathbf{n}}^{2}} \operatorname{Pf}[\mathcal{A}]\right)$, and $\operatorname{Pf}[\mathcal{A}]$ is given in Eq. (7). This definition of $n$ is more convenient than $\partial \ln Z / \partial \mu$, and the two definitions agree when either expression is small.

To compute the density $n$ requires a full-scale unquenched simulation, which is beyond the scope of this paper. However it is instructive to determine $n$ in the leading semi-classical (mean-field) approximation. We compute the effective potential obtained by integrating out the fermions, assuming a classical background $\langle\varphi\rangle$ field; we then determine $\langle\varphi\rangle$ to be the value that minimizes the effective potential.

Setting $J=0$ in Eq. (7) (and dropping the sign $\sigma$ ) we

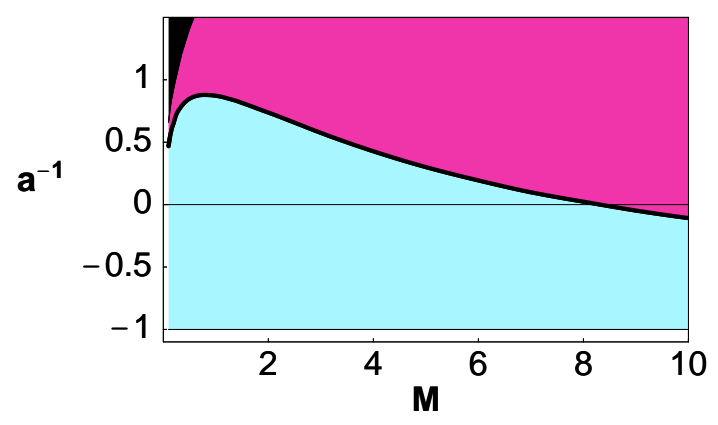

FIG. 1: The density phase diagram in the $\left(M, a^{-1}\right)$ plane at $\mu=0$ in the mean-field approximation for $M \geq 0.1$. The curve corresponds to a first order phase transition in the expectation value of the fermion number density $n$, with $n=0$ in the lower region and $n=O(1)$ in the upper region. The desired continuum physics only exists in the lower phase. The black region in the upper left corner is inaccessible for real scalar-fermion coupling.

can compute $\operatorname{Pf}[\mathcal{A}]$ exactly for constant $\varphi$ :

$$
\begin{aligned}
\operatorname{Pf}[\mathcal{A}] & =\operatorname{det} \tilde{K}^{\dagger} \tilde{K} \\
& =\prod_{\left\{\hat{p}_{i}\right\}}\left[\left(1+\Delta_{\mathbf{p}} / M\right)^{N_{\tau}}+e^{N_{\tau} \mu}(1+\varphi)^{N_{\tau}}\right]^{2} \\
& \equiv e^{-N_{\tau} N_{s}^{3} \mathcal{V}_{1}(\varphi)} \\
\mathcal{V}_{1}(\varphi) & =-\frac{2}{N_{s}^{3}} \sum_{\left\{\hat{p}_{i}\right\}}\left[\ln \left(1+\frac{\Delta_{\mathbf{p}}}{M}\right)+\frac{\ln \left(1+\left(\xi_{\mathbf{p}}(1+\varphi)\right)^{N_{\tau}}\right)}{N_{\tau}}\right] .
\end{aligned}
$$

In the $N_{\tau}, N_{s} \rightarrow \infty$ limit (large volume, zero temperature) this becomes

$$
\begin{aligned}
\mathcal{V}_{1}(\varphi)=-2 \int_{B Z} \frac{d^{3} \mathbf{p}}{(2 \pi)^{3}}\left[\ln \left(1+\Delta_{\mathbf{p}} / M\right)\right. \\
\left.+\ln \left(\xi_{\mathbf{p}}(1+\varphi)\right) \theta\left(\xi_{\mathbf{p}}(1+\varphi)-1\right)\right] .
\end{aligned}
$$

The momentum integration in the above expression is over the Brillouin zone, $p_{i} \in(-\pi, \pi]$. The first term in $\mathcal{V}_{1}$ is just the contribution from free fermions; the second term is the quantum correction to the tree level potential, $\mathcal{V}_{0}(\varphi)=\frac{1}{2} m^{2} \varphi^{2}$. In the mean-field approximation the expectation value $\langle\varphi\rangle$ is given by the minimum of the effective potential $\mathcal{V}_{\text {eff }}(\varphi)=\left(\mathcal{V}_{0}+\mathcal{V}_{1}\right)$

The effective potential yields the $\mu=0$ phase diagram displayed in Fig. 1] The horizontal axis is the fermion mass $M$, while the vertical axis is the inverse scattering length, $a^{-1}$. Moving up the vertical axis corresponds to increasing the strength of the two-body attraction, and $a^{-1} \geq 0$ describes systems with a two-body bound state. The curve corresponds to a jump in the fermion density from zero in the lower region, to $O(1)$ in the upper region. We see that there is a region far from the phase transition for $M \sim 1$ that allows small $a^{-1}$ of 


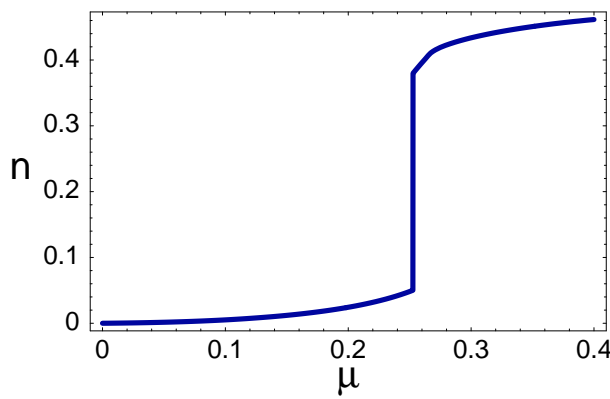

FIG. 2: Density $n$ as a function of $\mu$ for $M=1$ and $a \rightarrow \infty$. This figure exhibits a first order phase transition as a function of $\mu$. The small $\mu$ phase is where a continuum limit is defined.

either sign. Thus one may expect to be able to perform simulations of fermions at finite density, where both the interparticle spacing $n^{-1 / 3}$ and scattering length $|a|$ are large compared to the lattice spacing, without requiring $|\eta|$ to be small.

If one were to plot the chemical potential $\mu$ along a third axis of the phase diagram, then the critical line we have displayed would become a critical surface. In Fig. 2 we show the fermion number density $n$ plotted as a function of $\mu$, for fixed $M=1$ and $a^{-1}=0$. One sees that there is a regime where $n$ grows slowly with $\mu$, but that at a critical chemical potential, the fermion density jumps discontinuously as the trajectory crosses the critical surface. We see that at least in the meanfield approximation, there is a large region in parameter space where we can define continuum theories with any value of $\eta \equiv\left(a n^{1 / 3}\right)$ we desire.

One of the first tasks of a full-fledged simulation will be to determine the analog of Fig. 1 beyond the mean field approximation by evaluating the expression in Eq. (10) and to show that a region in parameter space with an interesting continuum limit persists. An interesting calculation to pursue subsequently would be to map out the critical temperature for the superfluidity phase transition with order parameter $\langle\psi \psi\rangle$ as a function of the dimensionless quantity $\eta$. To do this one would need to simulate the system at finite source $J$, and extrapolate to the $J=0$, infinite volume limit.

\section{DISCUSSION}

There are numerous ways in which the lattice theory we have presented may be modified or extended without sacrificing its crucial property of positivity. Several obvious possibilities are: (i) to include a kinetic term for the $\varphi$ field, allowing for attractive fermion interactions extended in space (for example, a Yukawa interactions for the simulation of neutron matter, for which $p$-wave interactions are expected to be important 10]); (ii) the $\varphi$ field could be given derivative and spin-dependent couplings, perhaps allowing one to include the contributions from the two-body effective range or scattering in higher partial waves; (iii) more flavors can be introduced, which could provide an opportunity to study the importance of three-body contact interactions and the possibility of renormalization group limit cycles [11, 12, 13, 14].

\section{Acknowledgments}

We wish to thank P. Bedaque, G. Bertsch, E. Braaten, J. Carlson, R. Furnstahl, S. Reddy, S. Sharpe, D. Son and L. Yaffe for helpful communications. J.W.C. thanks the INT at the U. of Washington and the INFN at the U. of Rome La Sapienza for hospitality. J.W.C. is supported in part by the National Science Council of ROC and the U.S. department of energy under grant DOE/ER/40762213. D.B.K was supported in part by DOE grant DEFGO3-00ER41132.
[1] S. Inouye, M. Andrews, J. Stenger, H.-J. Miesner, D. Stamper-Kurn, and W. Ketterle, Nature 392, 151 (1998).

[2] P. Courteille, R. Freeland, D. Heinzen, F. van Abeelen, and B. Verhaar, Phys. Rev. Lett. 81, 69 (1998).

[3] J. L. Roberts, N. R. Claussen, J. P. Burke, Jr., C. H. Greene, E. A. Cornell, and C. E. Wieman, Phys. Rev. Lett. 81, 5109 (1998).

[4] B. G. Levi, Physics Today 56, 18 (2003).

[5] J. Carlson, S. Y. Chang, V. R. Pandharipande, and K. E. Schmidt, Phys. Rev. Lett. 91, 50401 (2003), physics/0303094.

[6] P. Hasenfratz and F. Karsch, Phys. Lett. B125, 308 (1983).

[7] J. B. Kogut, D. K. Sinclair, S. J. Hands, and S. E. Morrison, Phys. Rev. D64, 094505 (2001), hep-lat/0105026.
[8] S. Hands, B. Lucini, and S. Morrison, Phys. Rev. D65, 036004 (2002), hep-lat/0109001.

[9] D. B. Kaplan, M. J. Savage, and M. B. Wise, Nucl. Phys. B478, 629 (1996), nucl-th/9605002.

[10] P. F. Bedaque, G. Rupak, and M. J. Savage (2003), nuclth/0305032.

[11] P. F. Bedaque, H. W. Hammer, and U. van Kolck, Phys. Rev. Lett. 82, 463 (1999), nucl-th/9809025.

[12] S. R. Beane, P. F. Bedaque, L. Childress, A. Kryjevski, and J. McGuire, Phys. Rev. A64, 042103 (2001), quantph/0010073.

[13] S. D. Glazek and K. G. Wilson, Phys. Rev. Lett. 89, 230401 (2002), hep-th/0203088.

[14] M. Bawin and S. A. Coon (2003), quant-ph/0302199. 